\title{
Monitor a Driver Behavior by ECG Measurement
}

\author{
Ching-Sung Wang, Yin-Cheng Huang, Teng-Wei Wang, and Si-Huei Lee
}

\begin{abstract}
This study focus for a driver in day care, real-time ECG monitoring is required, because symptoms of cardiac arrhythmia and hypoxia can occur unexpectedly. To prevent any cardiovascular problems unknown to a driver and to enhance care service and quality, in this system, the front-end sensor extracts the measured physiological signals from a driver's hand in the vehicle, subsequently wirelessly transmitting these signals to the Android system for processing. This research proposes a continue ECG monitoring system for transmitting ECG signals to Android mobile phones or tablets through Bluetooth transmission in vehicles. These signals are then uploaded to a cloud database, enabling health care physicians and nurses to instantly monitor and access ECG data. The system can effectively reduce the rate of accidents caused by drivers.
\end{abstract}

Index Terms-ECG, driver behavior, real-time, Android, cloud database.

\section{INTRODUCTION}

Numerous studies have investigated home care systems [1]-[4], with the integration and advancement of wireless communication and embedded microcontrollers prompting the development of wireless sensor networks. In recent years, international and domestic studies have vigorously developed telemedicine and wireless healthcare systems. Several studies have focused on hardware design, including humanized wireless sensors that could continually detect signals. Therefore, the development of wireless home care systems has attracted increasing attention.

Android smart systems and sensors typically employ audio cables to input and store signals. This study sets up a driver's ECG monitoring system in which microprocessors are used to process signals. The processed signals are then transmitted using Bluetooth modules to the user interface on an Android phone or tablet for immediate processing and displaying of electrocardiogram (ECG) signals, heart rhythms, and GPS positioning. Finally, these data are transmitted through a wireless network to the cloud database of a care center to facilitate immediate and excellent health care service [5]. They can be used for the establishment of a driver behavior model and can reduce the possibility of a car accident.

\section{System ARCHITECTURE}

Manuscript received June 12, 2018; revised November 12, 2018

Ching-Sung Wang is with the Department of Electronic Engineering, Oriental Institute of Technology, Taiwan (e-mail: ff020@mail.oit.edu.tw)

Ying-Cheng Huang is with the Department of Electric Engineering, National Chiao Tung University, Taiwan.

Teng-Wei Wang is with the Department of Thoracic Surgery, Zhangzhou Third Hospital, Zhangzhou City, Fujian Province, China.

Si-Huei Lee is with the Department of Physical Medicine and Rehabilitation, Taipei Veterans General Hospital, Taipei, Taiwan.
The system proposed in this study comprises three sections: driver's measurement end, human machine interface, and care center (Fig. 1). In this system, the front-end sensor extracts the measured physiological signals from a driver's hand in the vehicle, subsequently wirelessly transmitting these signals to the Android system for processing. The processed signals are then sent through a wireless network to the telecare database where they are stored. This system is capable of synchronously transmitting users' ECG signal through a wireless network to the cloud server, thereby facilitating continuous precise recording. Caregivers are concurrently provided with comprehensive data, enabling drivers to receive immediate and adequate care. Fig. 1 shows the system ECG measurements.

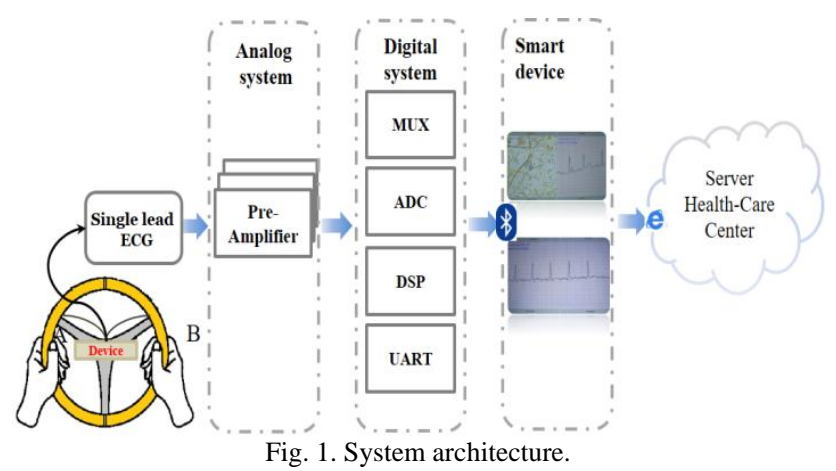

\section{ANAlOG SySTEM DESIGN}

The system proposed in this study comprises three sections: driver's measurement end, human machine interface, and care center (Fig. 1). In this system, the front-end sensor extracts the measured physiological signals from a driver's hand in the vehicle, subsequently wirelessly transmitting these signals to the Android system for processing. The processed signals are then sent through a wireless network to the telecare database where they are stored. This system is capable of synchronously transmitting users' ECG signal through a wireless network to the cloud server, thereby facilitating continuous precise recording. Caregivers are concurrently provided with comprehensive data, enabling drivers to receive immediate and adequate care. Fig. 1 shows the system ECG measurements.

In this study the input end of physiological signal measurement can be quickly integrated with the steering wheel of an automobile through a conductive fiber cloth [6]. As shown in (Fig. 1), the steering wheel includes two yellow sections on both sides (Side A and Side B), which have the features of conductivity, electromagnetic wave protection, and plasticity. The average resistance value of the conductive cloth is about $0.03 \Omega$ (contact impedance). An ideal magnifier features infinite input impedance, but the equivalent impedance generated by the friction or touch between skin and the conductive cloth will result in an electric potential 
difference on both ends and lead to an increase in the number of common-mode signals.

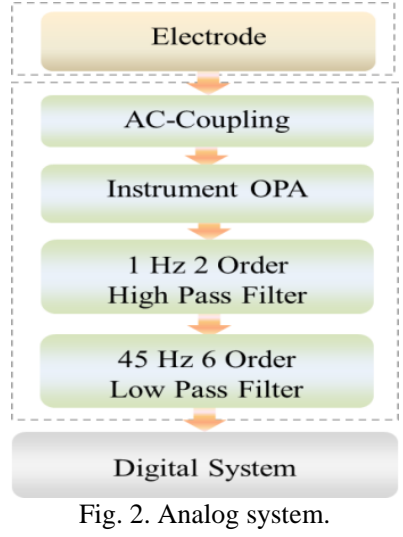

Fig. 2 shows the hardware architecture of the system. The miniaturized ECG monitoring developed in this study has a newly-designed AC coupling circuit that needs two-electrode technology to protect users and to eliminate the non-circuit feedback of the earth electrode [7]. There is a single-source power supply system, and the electric circuit is simplified to reduce the area of the circuit and lower power consumption. It can also be widely used in telecare. Signals are sent from the differential measurement point (Fig. 3) to AC-Coupling, and the frond-end maximizer adopts the AC-Coupling circuit, which features high input impedance, a high CMRR value, little noise, low deviation, protection for a circuit, and the elimination of the $60 \mathrm{~Hz}$ noise from the human body. It magnifies small ECG signals by 1,000 times at once and captures signals over $1 \mathrm{~Hz}$. Signals below $1 \mathrm{~Hz}$ are filtered through the second-order high pass filter, and the cut-off frequency of the six-order low pass filter is set below $45 \mathrm{~Hz}$. Through the High Pass Filter and the Low Pass Filter, the frequency is set between $1 \mathrm{~Hz}$ and $45 \mathrm{~Hz}$, and the original $60 \mathrm{~Hz}$ noise in the human body is filtered as shown in (Fig. 4).

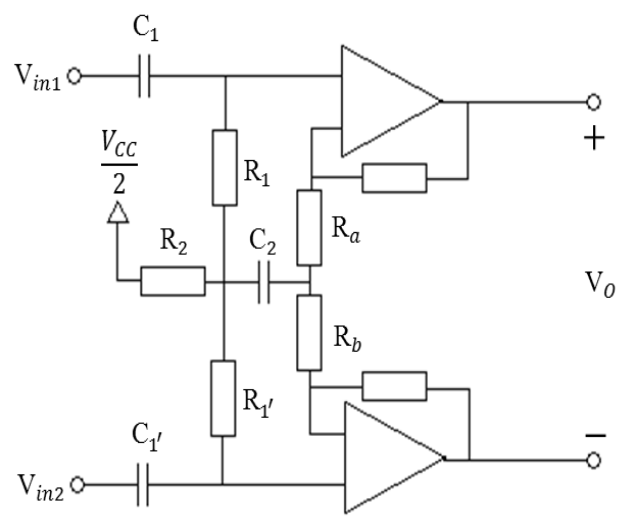

Fig. 3. Differential frond-end AC-coupling circuit.

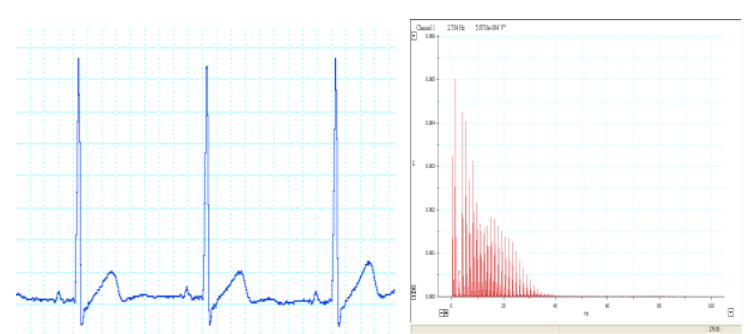

Fig. 4. ECG measurement signal and spectrum.

\section{ANAlog System DESIGN}

Fig. 5 illustrates, the proposed system, which is a multichannel signal system that measures discrete signals sampled from continuous signals. Therefore, the multichannel scan sampling device of the microprocessor, multichannel buffer, AD converter, and DSP are used in the digital system.

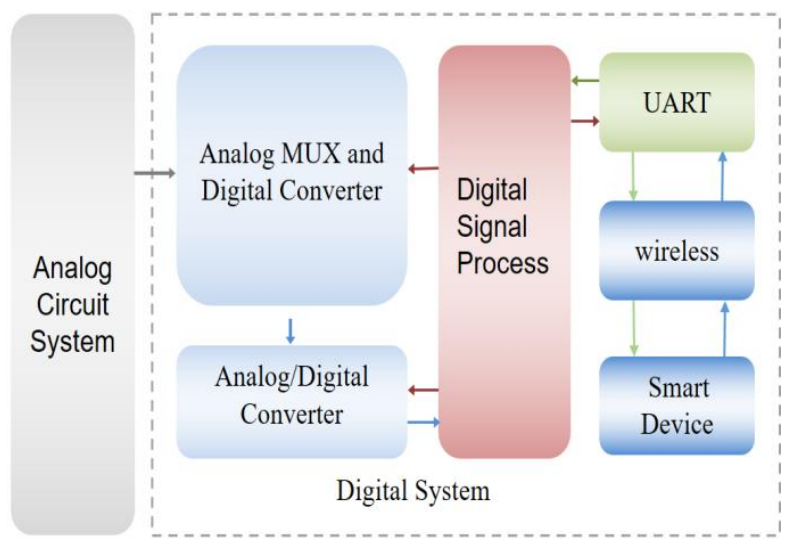

Fig. 5. Sensor digital system.

In the frequency bandwidth of an ideal filter, it is reserved without a reduction; on the contrary, signals at the zero point of the reduction curve will decrease exponentially. Hence, the filter needs to filter the frequency range of external noise $(60 \mathrm{~Hz})$, and the frequency of sampling needs to be at least 200 Samples/s. However, the analog filter is unable to resemble the ideal design in reality, and so it is necessary to re-filter noise according to the sampling rate. This study uses MATLAB TOOL to stimulate a low frequency response as shown in (Fig. 6).

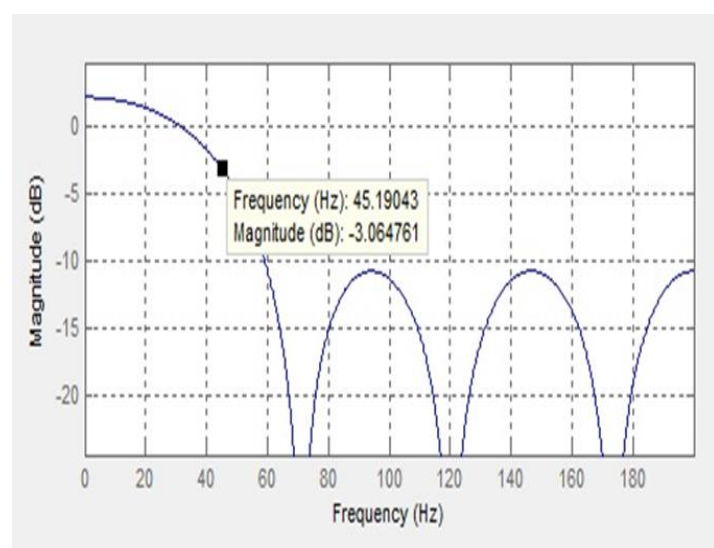

Fig. 6. The $45 \mathrm{~Hz}$ low-frequency filter stimulates a response.

\begin{tabular}{|c|c|c|c|}
\hline \multicolumn{3}{|c|}{ TABLE I: TRANSMISSION FORMAT } \\
\hline Head & ECG Data & Temperature. & End \\
\hline 1 Byte & 400 Byte/s & 2 Byte/s & 1 Byte \\
\hline
\end{tabular}

Bluegiga WT12 is utilized on the wireless ends. Featuring integrated antennas, it meets the criteria for an enhanced data rate (EDR), with a maximum data rate of $2-3 \mathrm{Mbps}$ and a maximum transmission power of $4 \mathrm{dBm}$. The physiological information is sent and transmitted through the Bluetooth module. The receiving end is an Android smartphone or touch pad, which receives and processes the transmission format, as shown in (Table I). 


\section{SMART DeVICE System}

The user end is an Android smartphone or touch pad, as shown in (Fig. 7), which displays the GPS map and the location, the ECG wave form, and heart rate (HR) wave computation. It can be divided into six parts: (a) User Interface Activity, (b) Bluetooth Receiving Service, (c) Surface View High-speed Mapping, (d) Signal-processing Service, (e) Information Uploading Service, and (f) Information Exchange Queue. The user operation is a graphic one, and relevant information is shown in a written form. The positioning system uses Google Map function information and the fore-end sensor to achieve real-time updating and recording every second.

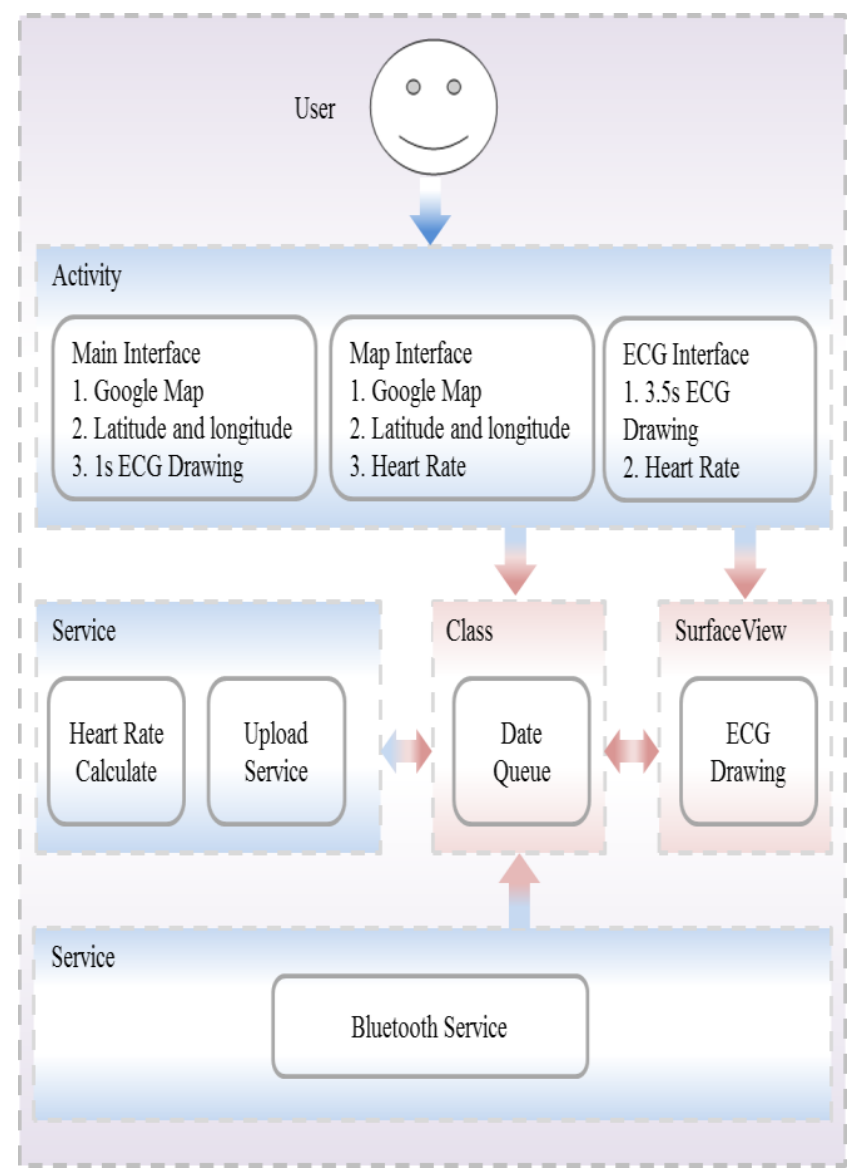

Fig. 7. User device system.

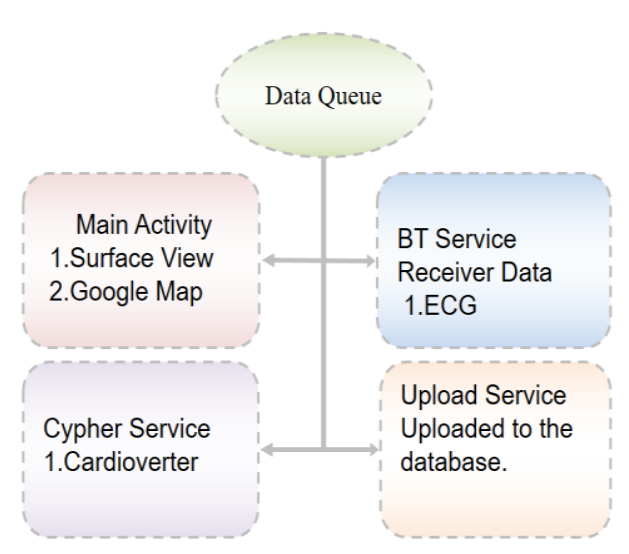

Fig. 8. Data exchange architecture.

There is a large quantity of ECG information, and so the ECG interface adopts the Activity interface embedded with the exclusive View-layer mapping from the high-speed mapping SurfaceView. It aims to provide a thread to draw graphs and can set the drawing rate itself. Categorization provides a thread for application programs to draw graphs, and so the Frame Per Second (FPS) of the SurfaceView category can be $34 \mathrm{fps}$, and the computation thread, the Bluetooth receiving thread, and the uploading thread can be established. The information is received by Bluetooth and then temporarily stored in the information queue (Fig. 8) for other executions.

The real-time HR algorithm from the system must be applicable to the Android system and must operate in the core virtual system Dalvik Virtual Machine of Linux. Therefore, the time and space complexity of the algorithm should not be excessive. To reduce the baseline transfer and the interference of external noise, it is necessary to divide the HR algorithm into several steps. In this system, the real-time ECG algorithm starts with time domain analysis, and the Slope Vector Waveform (SVW) of the QRS wave is used for the analysis [8], as SVW can be employed for an effective real-time test on the RS wave, can filter noise and other ECG elements, and can quickly and accurately detect the location of the $\mathrm{R}$ wave [9], [10], as shown in (Fig. 9). As mentioned above, the algorithm is divided into the following steps: (1) computation of the threshold value, (2) baseline processing, (3) smoothing, (4) SVW processing, (5) the test on R wave, and (6) HR computation.

The definition of threshold value is highly important in the ECG test. An effective threshold value can filter the interference caused by myoelectricity and external noise, while a poor threshold value increases the error rate in the test. This study considers the difference in the interference caused by different users and environments in the ECG, and so the threshold value must change with external factors and is thus called the self-adaptive threshold value, which can adjust itself according to a change in external environment or measurement [11]. When signals are magnified in display, a serious baseline transfer is likely to happen due to the excessive amplitude of vibration. To reduce the baseline transfer, the Simple moving Average (SMA) is used to reduce the ECG to the level of $0 \mathrm{~V}$. At the same time, the source information of ECG is set as " $y$ "; the frequency of sampling as " $f s$ "; and the average moving baseline as "BL". The equation of baseline processing is as follows:

$$
\mathrm{BL}=\frac{1}{f_{S}} \sum_{i=-\infty}^{\infty} y_{i}
$$

There is a minor wave form in the electro cardio-measurement. The wave form is likely to cause a wrong judgment and increase difficulty in the test on the $\mathrm{R}$ wave. To reduce the mistake, priority is given to the smoothing of wave forms. In this study the system resource and curve need to be taken into account, as excessive smoothing results in distortion and eliminates the features of the ECG. In this case, the three-point moving average is used for smoothing, which can filter low-frequency noise and enhance the accuracy of testing.

The information source of the ECG is marked as " $y$ "; the information at each point as " $i$ "; and each set of information after smoothing as Smoothi. The equation is: 


$$
\operatorname{Smooth}_{i}=\frac{y_{i-1}+y_{i}+y_{i+1}}{3}
$$

When the range (0.06s $\sim 0.12 \mathrm{~s})$ of the QRS compound wave, a periodical parameter of the ECG, is divided by the frequency of sampling, the interval of the QRS wave can be obtained. The equation is:

$$
Q R S_{\text {point }}=s / \frac{1}{f_{s}}
$$

The SVW of the location of the R wave must be continually higher than or equivalent to the threshold value " $n$ " and must be the maximum (peak value) of the wave form. The equation of the threshold " $n$ " is:

$$
n=\sqrt{Q R S_{\text {MaxPoint }}+Q R S_{\text {MaxPoint }}} / 2
$$

For computation of the threshold value, the sampling frequency is set as "fs" and the number of sampling as "C". The equation of the self-adaptive threshold is:

$$
S A T_{c}=\frac{1}{f s} \sum_{c_{i}=-\infty}^{\infty} \frac{y c_{i+1}-y c_{i}}{t c_{i+1}-t c_{i}}
$$

\section{DATA ANALYSIS}

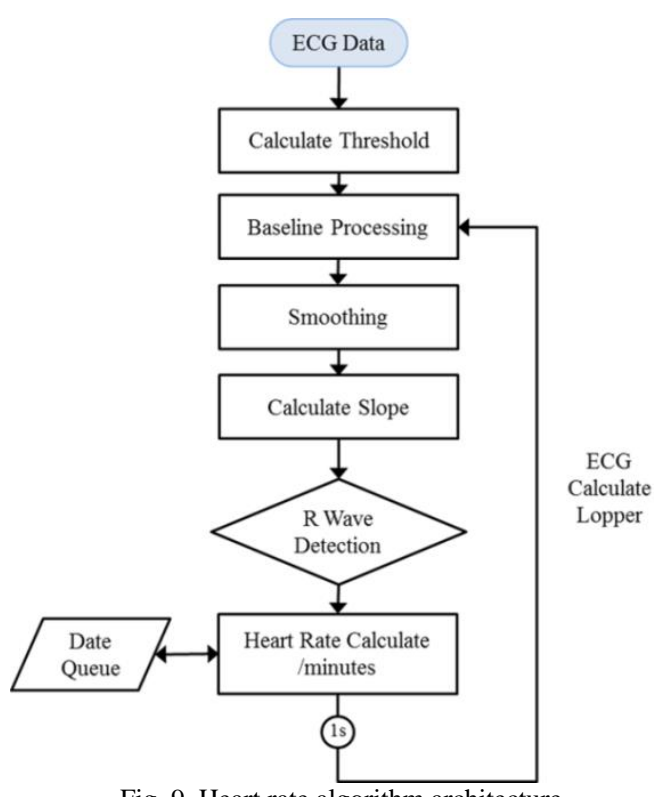

Fig. 9. Heart rate algorithm architecture.

For the actual measurement of the ECG signals of the human body, GE Datex-Ohmeda, an advanced multi-functional physiological monitor, is used to measure the ECG and HR. The three-lead ECG and $\mathrm{SpO} 2$ and the self-made single-lead ECG measurement sensor are utilized for measurement comparison. According to the comparison between the self-made physiological signal measurement system and the advanced multi-functional physiological monitor, the latter can accurately measure the features of electrocardio signals, display wave forms, and calculate HR.
According to the data in Table II, there is an error in the HR range of 50 100BPM on average, with 30 subjects in the actual measurement.

TABLE II: TRANSMISSION FORMAT

\begin{tabular}{lll}
\hline & System Spec & This Study \\
\hline Pre-Amp. CMRR(dB) & $80 \mathrm{~dB}$ & More than $130 \mathrm{~dB}$ \\
High Pass Filter & $0.05 \mathrm{~Hz}$ & $0.05 \mathrm{~Hz}$ \\
Low Pass Filter & $50 \mathrm{~Hz}$ & $45 \mathrm{~Hz}$ \\
Sample Frequency & $300 \mathrm{~Hz}$ & $400 \mathrm{~Hz}$ \\
Wireless Frequency & $2.36-2.4 \mathrm{GHz}$ & $2.4 \mathrm{GHz}$ \\
BPM Error & 1 & 1 \\
\hline \hline
\end{tabular}

\section{DATA ANALYSIS}

The electrocardio features can be used to detect a R-R wave per second. When a driver drives a car with one hand on the steering wheel, the system can recognize the situation and record it or remind the driver to drive with both hands, as shown in (Fig. 10).

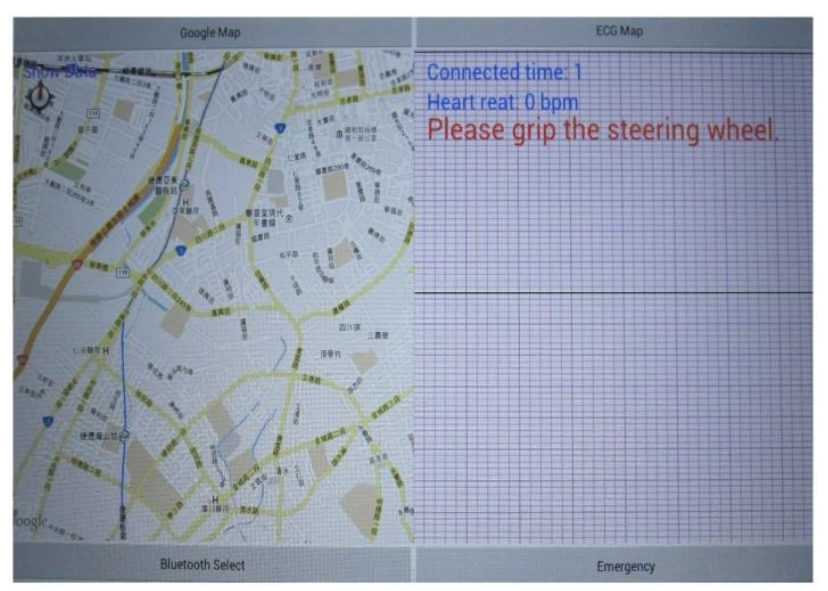

Fig. 10. Main interface.

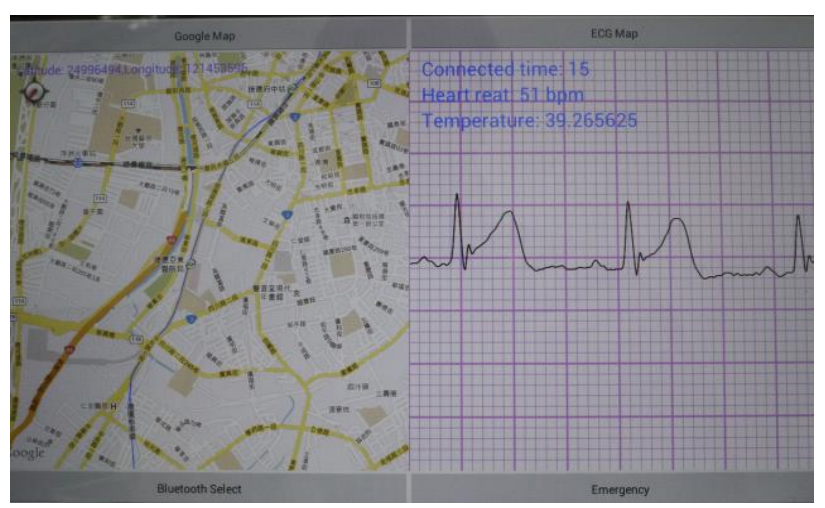

Fig. 11. Main interface with information.

\section{CONCLUSION}

This study has designed a miniature ECG and body temperature monitoring system. The hardware comprises an AC-coupling circuit and filter; the software includes digital filtering, wireless transmission, heart rhythm algorithm, GPS positioning, and ECG display interface. The system can enable 24-hour continuous monitoring and immediate positioning, accurately calculate heart rhythms, display ECG signals, and synchronously upload data to a cloud database. Without using any electrical wires, this lightweight and 
portable system can be attached to users' chest to continuously monitor them and to provide them with excellent care services and data access. Moreover, this study can be used to establish a driver behavior model and reduce the possibility of a car accident.

\section{REFERENCES}

[1] R. Mietzner et al., "Horizontal and vertical combination of multi-tenancy patterns in service-oriented applications," Enterprise Information Systems, vol. 5, no. 1, pp. 59-77, 2011.

[2] L. Xu, "Enterprise systems: state-of-the art and future trends," IEEE Transactions on Industrial Informatics, vol. 7, no. 4, pp. 630-640, 2011.

[3] L. Duan et al., "Healthcare information systems: Data mining methods in the creation of a clinical recommender system," Enterprise Information Systems, vol. 5, no. 2, pp. 169-181, 2011.

[4] C.-S. Wang, "The implementation of a tele-homecare system with service oriented architecture and HL7 message transmission standard," American Journal of Public Health Research, 2013, vol. 1, no. 1, pp. 18-26.

[5] Y.-C. Su, H. Chen et al., "Wireless ECG detection system with low-power analog front-end circuit and bio-processing ZigBee firmware," in Proc. 2010 IEEE International Symposium on Circuits and Systems (ISCAS), 2010

[6] W. Zhang et al., "Carbon based conductive polymer composites," Journal of Materials Science, May 2007, vol. 42, issue 10, pp. 3408 3418.

[7] C.-S. Wang, "A new AC-coupled amplifier for portable ECG without reference electrode," J. Computer and Electric Eng., Jun. 2013.

[8] H. Y. Yang and R. Sarpeshkar, "A time-based energy-efficient analog-to-digital converter," IEEE J. Solid-State Circuits, vol. 40, no. 8, 2005.

[9] X. M. Xu and Y. Liu, "ECG QRS complex detection using slope vector waveform (SVW) algorithm," in Proc. the 26th Annual International Conference of the IEEE EMBS, San Francisco, CA, USA, September $1-5,2004$.

[10] S. Karpagachelvi, M. Arthanari, and M. Sivakumar, "ECG feature extraction techniques - A survey approach," International Journal of Computer Science and Information Security (IJCSIS), vol. 8, no. 1, April 2010.

[11] J. Willis et al., "A real-time QRS detection algorithm," IEEE Transactions on Biomedical Engineering, vol. BME-22, pp. 230-236, March 2005 .

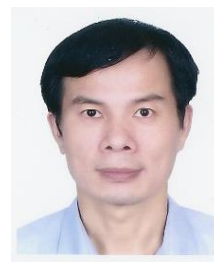

Ching-Sung Wang was born in Taipei, Taiwan. He received the electronic engineering M.S. degree from the Chung Yuan Christian University, and the Ph.D. degree in computer aided design from the National Taiwan University, in 1987 and 1998, respectively. And major analog integrated circuit design. $\mathrm{He}$ is currently a professor of Department of Electronic Engineering at the Oriental Institute of Technology, New Taipei City, Taiwan. He current interests are to develop health-care information system and biomedical instrumentation.

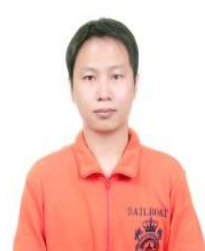

Yin-Cheng Huang was born in Taiwan. He received the information and communication engineering M.S degree from the Department of Electronic Engineering at the Oriental Institute of Technology, in 2012. And major analog integrated circuit design. Currently studying for a doctoral degree at the Institute of Electrical Engineering, National Chiao Tung University. He current interests are to develop internet of things information system and biomedical instrumentation.

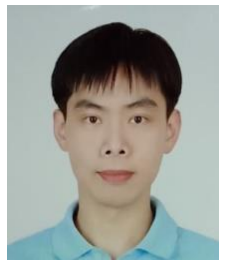

Teng-Wei Wang was born in Taipei, Taiwan. He found himself interest was in bio-chemistry and medicine. He graduated with his medical degree of MB $\mathrm{ChB}$ in 2008. Following this he did one year's post-graduated training in his University Hospital. $\mathrm{He}$ is currently working in Department of Thoracic Surgery, ZhangZhou Third Hospital, ZhangZhou City, Fujian Province, China. He still want to promote himself in the field of research, especially prevention or prognosis factors of lung cancer or esophageal cancer, which is his profession related as a thoracic surgeon.

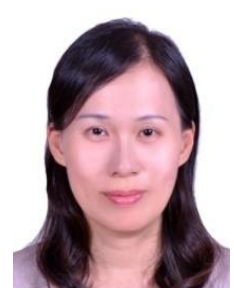

Si-Huei Lee was born in Taichung, Taiwan. She received the bachelor degree in medicine from the China Medical University, and the Ph.D. degree in Health and Welfare from the Japan Niigata University of Health and Welfare in 1999 and 2018, respectively. She is currently an attending physician of Taipei Veterans General Hospital, and also a principle investigator of intelligent medical rehabilitation laboratory at the same institute. She current interests are to develop health-care information system and smart rehabilitation instrumentation. 